\title{
Protective effect of thymoquinone improves cardiovascular function, and attenuates oxidative stress, inflammation and apoptosis by mediating the PI3K/Akt pathway in diabetic rats
}

\author{
HUI LIU ${ }^{1}$, HONG-YANG LIU ${ }^{2}$, YI-NONG JIANG ${ }^{3}$ and NAN LI ${ }^{1}$ \\ ${ }^{1}$ Department of Emergency; ${ }^{2}$ Heart Intensive Care Unit; ${ }^{3}$ Department of Cardiology, \\ The First Affiliated Hospital of Dalian Medical University, Dalian, Liaoning 116011, P.R. China
}

Received April 20,2015; Accepted December 2, 2015

DOI: $10.3892 / \mathrm{mmr} .2016 .4823$

\begin{abstract}
Thymoquinone is the main active monomer extracted from black cumin and has anti-inflammatory, antioxidant and anti-apoptotic functions. However, the protective effects of thymoquinone on cardiovascular function in diabetes remain to be fully elucidated. The present study aimed to investigate the molecular mechanisms underling the beneficial effects of thymoquinone on the cardiovascular function in streptozotocin-induced diabetes mellitus (DM) rats. Supplement thymoquinone may recover the insulin levels and body weight, inhibit blood glucose levels and reduce the heart rate in DM-induced rats. The results indicated that the heart, liver and lung to body weight ratios, in addition to the blood pressure levels, were similar for each experimental group. Treatment with thymoquinone significantly reduced oxidative stress damage, inhibited the increased endothelial nitric oxide synthase protein expression and suppressed the elevation of cyclooxygenase-2 levels in DM-induced rats. In addition, thymoquinone significantly suppressed the promotion of tumor necrosis factor- $\alpha$ and interleukin- 6 levels in the DM-induced rats. Furthermore, administration of thymoquinone significantly reduced caspase- 3 activity and the promotion of phosphorylated-protein kinase B (Akt) protein expression levels in DM-induced rats. These results suggest that the protective effect of thymoquinone improves cardiovascular function and attenuates oxidative stress, inflammation and apoptosis by mediating the phosphatidylinositol 3-kinase/Akt pathway in DM-induced rats.
\end{abstract}

Correspondence to: Ms. Hong-Yang Liu, Heart Intensive Care Unit, The First Affiliated Hospital of Dalian Medical University, 193 Lianhe Road, Dalian, Liaoning 116011, P.R. China

E-mail: hongyangliuewd@163.com

Key words: thymoquinone, diabetic cardiovascular disease, cardiovascular functions, PI3K/Akt

\section{Introduction}

Diabetes mellitus (DM) is a type of chronic metabolic disease involving the interaction of genetic and environmental factors. It is characterized by dysinsulinism, insulin resistance (IR) and disorders of the blood sugar, lipid and protein metabolisms (1). An increase in the prevalence rate of DM in China is reported to be due to the economic development and increase in the average life expectancy (2). The first national census of DM demonstrated that the prevalence rate in China was $1 \%$ in 1979 and 2.02\% in 1989 (3). In addition, the prevalence rate of type-I DM was $0.00057 \%$ and type-II DM was $3.21 \%$ in 1996 (4). At present, there are 40 million patients with DM in China (4), however, in developed cities, such as Shanghai, the prevalence rate of DM may be as high as $10 \%$ (5). The number of obese individuals, particularly those suffering from abdominal obesity, has increased due to the change of living standards, resulting from the increased levels of sugar in their diet, smoking and alcohol consumption. Therefore, the higher prevalence rate of DM observed may be due to abdominal obesity, as it had been reported to be the basis of IR (6).

DM is characterized by chronic complications resulting in pain for the patients and a financial burden to their families (7). Diabetic cardiovascular disease (DCD) is one of the leading causes of death in patients with DM. The cardiovascular complications may occur at an early stage in DM and have a progressive development (8). The risk of stroke in patients with DM is 2 to 4 times higher compared with the risk of patients with cardiovascular diseases and without DM $(7,8)$. Furthermore, patients suffering from DM and myocardial infarction have reduced cardiac output compared with patients with myocardial infarction and without DM (7).

Thymoquinone is the main active monomer extracted from black cumin in Middle Eastern countries (9). A previous study demonstrated that thymoquinone acts as a tumor suppressor and inhibits the growth of various types of cancer cells (10). The aim of the present study was to identify whether the protective effect of thymoquinone improves cardiovascular function in diabetic rats and to explore its possible mechanisms. 


\section{Materials and methods}

Reagents. Streptozotocin (STZ) and thymoquinone (purity $>98 \%$ ) were purchased from Sigma-Aldrich (St. Louis, MO, USA). Malondialdehyde (MDA), superoxide dismutase (SOD), cyclooxygenase (COX)-2, tumor necrosis factor- $\alpha$ (TNF- $\alpha$ ) and interleukin-6 (IL-6) ELISA kits and caspase-3 activity kits were purchased from Jiancheng Biotech Co., Ltd. (Nanjing, China).

Animals. A total of 40 healthy male albino Wistar rats (6-8 weeks old; 230-300 g) were purchased from the Animal Experiment Center of Dalian Medical University (Dalian, China) and maintained under standard conditions $\left(22 \pm 2^{\circ} \mathrm{C}\right.$ with a 12-h dark-light cycle) and experiments were conducted in accordance with the Guide for the Care and Use of Laboratory Animals adopted by the Institutional Animal Care and Use Committee (11). All rats were fed with regular chow and given free access to water.

Model and grouping. All rats were randomly assigned into four groups ( $\mathrm{n}=10)$ : Sham group (Sham), thymoquinone group (THY), DM group (DM) and DM + thymoquinone group $(\mathrm{DM}+\mathrm{THY})$. STZ was dissolved in citrate buffer (Beijing Tiandz Biological Technology, Inc., Beijing, China; $60 \mathrm{mg} / \mathrm{kg}$ of body weight, $\mathrm{pH} 4.5$ ) and DM was induced by intravenous injection of STZ into the tail vein of the rats. The sham group was injected with the same volume of citrate buffer only, and the thymoquinone group was injected with $50 \mathrm{mg} / \mathrm{kg}$ thymoquinone for 30 days. The DM and DM + THY groups were injected with STZ + citrate buffer to induce DM. The DM group was then treated with citrate buffer only and the $\mathrm{DM}+\mathrm{THY}$ group was injected with $50 \mathrm{mg} / \mathrm{kg}$ thymoquinone for 30 days.

Biochemical and body weight measurements. Body weight was determined for all groups prior and subsequent to DM-induction and treatments. Blood samples were collected from the tail vein of rats and glucose levels were measured using the glucose oxidase method (Bpc BioSed Srl, Capena, Italy). A Glucose Oxidase ELISA kit (Elabscience Biotechnology Co., Ltd., Wuhan, China) and Insulin ELISA kit (Elabscience Biotechnology Co., Ltd.) were used to determine insulin levels. Insulin levels were measured using the Multiskan EX microplate photometer (Thermo Fisher Scientific, Inc., Waltham, MA, USA).

Tail-cuff blood pressure (BP) measurement. The CODA 8-channel high throughput non-invasive tail cuff blood pressure system (Kent Scientific Corporation, Torrington, CT, USA) was used to measure the BP of rats by determining the tail blood volume. All rats were placed into a designated platform maintained at $37^{\circ} \mathrm{C}$ and the system provided measurements of four BP parameters: Systolic, diastolic and mean BP and heart rate for $10 \mathrm{~min}$. The proximal occlusion cuff constricts the tail artery, while the distal cuff detects alterations in the tail artery volume when the blood flow resumes following the deflation of the occlusion cuff. Measurements of an average of three sessions, each consisting of 15 cycles, were used for statistical analysis.
Determination of oxidative stress, COX-2 and inflammation. Following thymoquinone treatment, blood was collected from the tail vein and centrifuged at $8,000 \mathrm{x} \mathrm{g}$, for $10 \mathrm{~min}$ at room temperature. The serum was used to measure the MDA, SOD, COX-2, TNF- $\alpha$ and IL-6 levels in accordance with the manufacturer's instructions (Jiancheng Biotech Co., Ltd.).

Determination of caspase-3 activity. Following thymoquinone treatment, rats were sacrificed by decollation under anesthesia (chloral hydrate; Sigma-Aldrich) and cardiac tissue specimens were collected and prepared in lysis buffer (Beijing Applygen Gene Technology Co., Ltd., Beijing, China). Samples were then centrifuged at $8,000 \times \mathrm{g}$ for $10 \mathrm{~min}$ at $4^{\circ} \mathrm{C}$ and protein concentration was determined with the $\mathrm{BCA}$ protein assay kit (Bio-Rad Laboratories, Ltd., Hemel Hempstead, UK) according to the manufacturer's instructions. The caspase-3 activity was blended by chromogenic caspase substrates and Ac-DEVD-pNA according to the manufacturer's instructions (Jiancheng Biotech Co., Ltd.), and measured at $405 \mathrm{~nm}$ with a Multiskan EX microplate photometer (Thermo Fisher Scientific, Inc.).

Western blot analysis. Following thymoquinone treatment, protein concentrations were determined as described above using the BCA protein assay kit (Bio-Rad Laboratories, Ltd.). Proteins $(50 \mathrm{mg})$ were separated by sodium dodecyl sulfate polyacrylamide gel (100 v; Beijing Tiandz Biological Technology, Inc.) electrophoresis in a $8-12 \%$ polyacrylamide gel, and transferred to polyvinyl difluoride membranes (Millipore, Billerica, MA, USA). Following blocking with 5\% non-fat milk, membranes were incubated overnight with monoclonal rabbit anti-endothelial nitric oxide synthase (eNOS; cat. no. 9572; 1:500, Cell Signaling Technology, Inc., Danvers, MA, USA), monoclonal rabbit anti-phosphorylated-protein kinase B (p-Akt; cat. no. 9271; 1:500; Cell Signaling Technology, Inc.) and monoclonal $\beta$-actin (cat. no. sc-1616; 1:500; Santa Cruz Biotechnology, Inc., Santa Cruz, CA, USA) primary antibodies. Membranes were then incubated with horseradish peroxidase-conjugated goat anti-biotin secondary antibody (cat. no. 7075; 1:500; Cell Signaling Technology, Inc.) for $2 \mathrm{~h}$ at room temperature, and proteins were visualized using Hybond enhanced chemiluminescence (Amersham International; GE Healthcare Life Sciences, Chalfont, UK). Protein expression was quantified using ImageJ software (version 1,37; National Institute of Health, Bethesda, MD, USA).

Statistical analysis. Data are presented as the mean \pm standard deviation. Statistical analysis was performed using analysis of variance, followed by a post hoc Newman-Keuls test (InStat software version 3.06; Graphpad Software, Inc., La Jolla, CA, USA). $\mathrm{P}<0.05$ was considered to indicate a statistically significant difference.

\section{Results}

Effect of thymoquinone treatment on the ratios of heart, liver and lung to body weight. The chemical structure of thymoquinone is presented in Fig. 1. As indicated in Fig. 2, the ratios of heart, liver and lung to body weight were not significantly different among the experimental groups. 
<smiles>CC1=CC(=O)C(C(C)C)=CC1=O</smiles>

Figure 1. Chemical structure of thymoquinone.

A

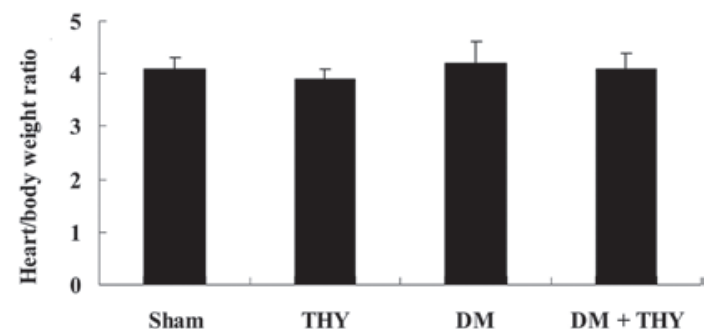

B

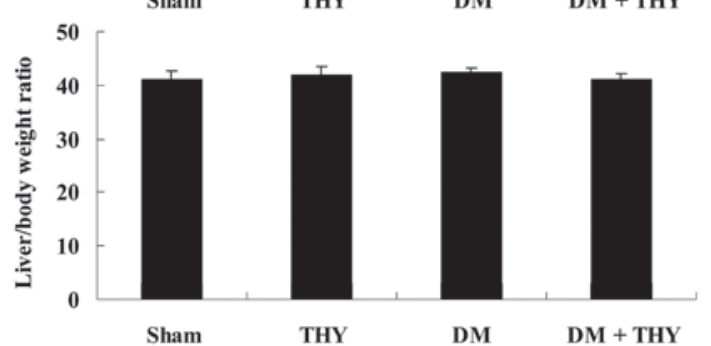

C

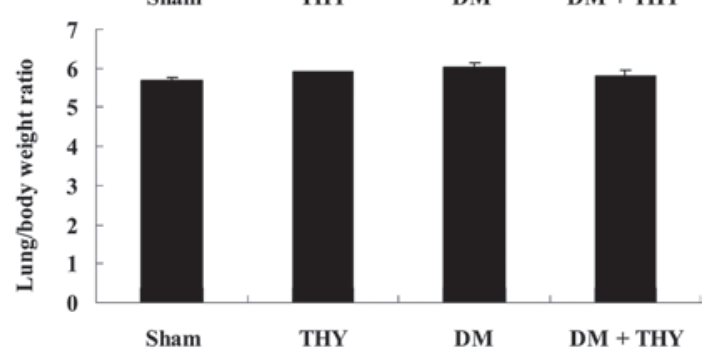

Figure 2. Effect of THY on body/weight ratio in the (A) heart, (B) liver and $(\mathrm{C})$ lung tissues in the sham, THY-treated, DM-induced and DM + THY-treated groups. THY, thymoquinone; DM, diabetes mellitus.

Effect of thymoquinone treatment on the insulin levels and body weight. As demonstrated in Fig. 3, the insulin levels and body weight in the DM-induced group were lower compared with the sham $(\mathrm{P}<0.01)$ and THY-treated groups. In addition, administration of thymoquinone resulted in an increase in the insulin levels and body weight of the DM + THY-treated group compared with the DM-induced group $(\mathrm{P}<0.01 ;$ Fig. 3$)$.

Effect of thymoquinone treatment on the blood glucose levels. As demonstrated in Fig. 4, the levels of blood glucose were significantly increased in the DM-induced groups compared with the sham $(\mathrm{P}<0.01)$ and THY-treated groups during the 30-day experiment. Administration of thymoquinone resulted in a reduction in the blood glucose levels of the DM-induced rats compared with the DM rats that had not received the treatment (P<0.01; Fig. 4).
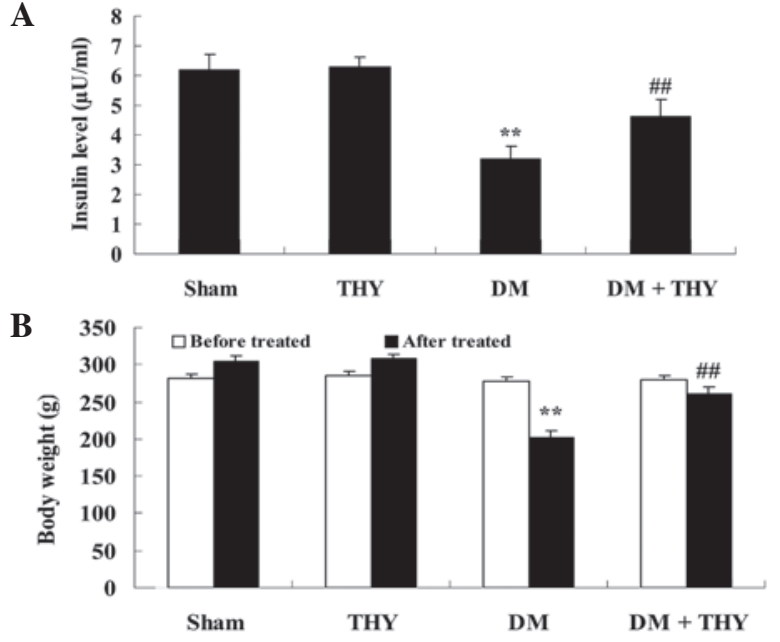

Figure 3. Effect of THY on (A) insulin levels and (B) body weight in the sham, THY-treated, DM-induced and DM + THY-treated groups. ${ }^{* *} \mathrm{P}<0.01$ vs. the sham group and ${ }^{\# \#} \mathrm{P}<0.01$ vs. the $\mathrm{DM}$-induced group. THY, thymoquinone; DM, diabetes mellitus.

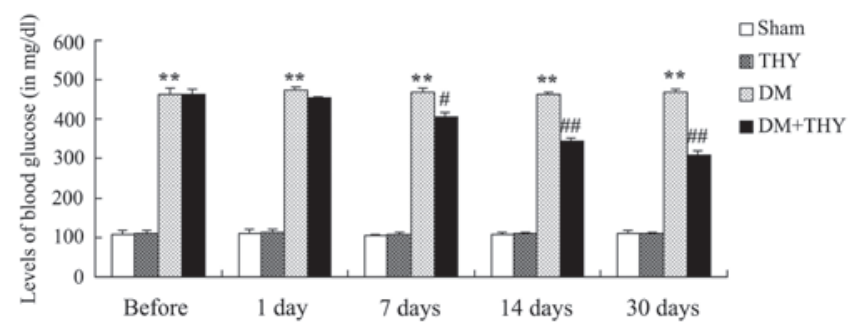

Figure 4. Effect of THY on the blood glucose levels the sham, THY-treated DM-induced and DM + THY-treated groups. ${ }^{* *} \mathrm{P}<0.01$ vs. the sham-treated group, ${ }^{\#} \mathrm{P}<0.05$ and ${ }^{\# \prime} \mathrm{P}<0.01$ vs. the DM-treated group. THY, thymoquinone; DM, diabetes mellitus.

A

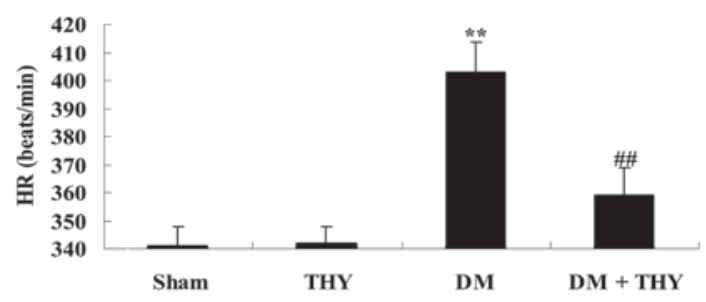

B

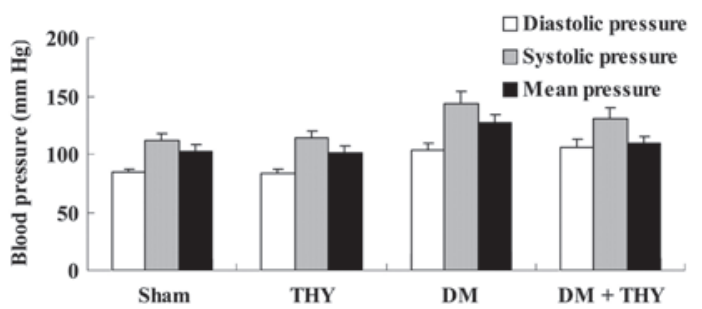

Figure 5. Effect of THY on (A) heart rate and (B) blood pressure in the sham, THY-treated, DM-induced and DM + THY-treated groups. ${ }^{* *} \mathrm{P}<0.01$ vs. the sham-treated group and ${ }^{\# \#} \mathrm{P}<0.01$ vs. the DM-treated group. THY, thymoquinone; DM, diabetes mellitus.

Effect of thymoquinone treatment on BP and heart rate. A significant increase in the heart rate of DM-induced rats was observed compared with the sham $(\mathrm{P}<0.01)$ and THY-treated 
$\mathbf{A}$

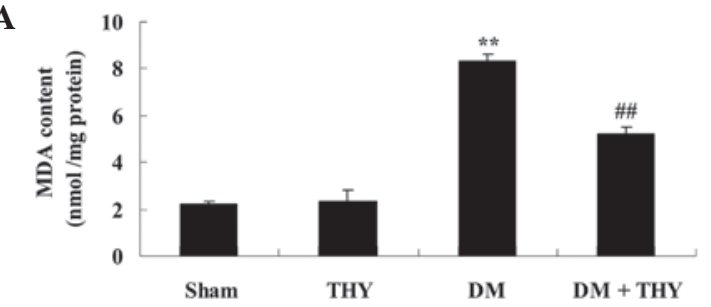

B

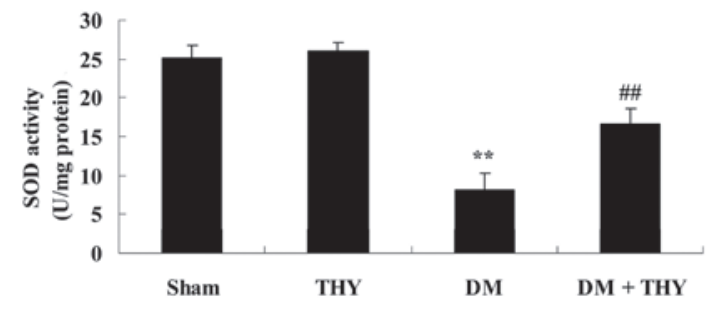

Figure 6. Effect of THY on the (A) MDA and (B) SOD content in the sham, THY-treated, DM-induced and DM + THY-treated groups. ${ }^{* *} \mathrm{P}<0.01$ vs. the sham-treated group and ${ }^{\# \#} \mathrm{P}<0.01$ vs. the DM-treated group. THY, thymoquinone; MDA, malondialdehyde; DM, diabetes mellitus; SOD, superoxide dismutase.

A

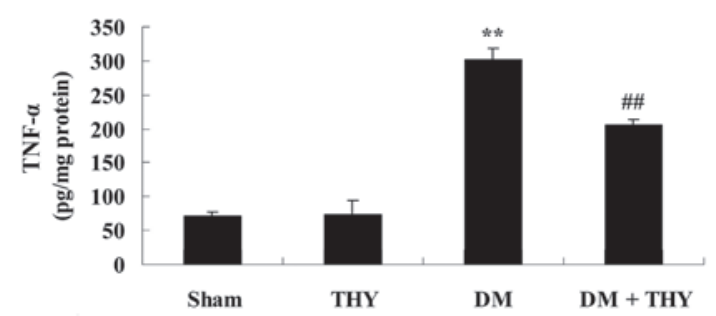

B

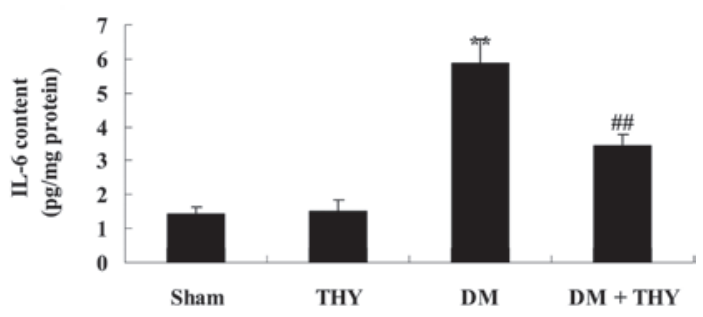

Figure 7. Effect of THY on the (A) TNF- $\alpha$ and (B) IL-6 level in the sham, THY-treated, DM-induced and DM + THY-treated groups. ${ }^{* *} \mathrm{P}<0.01$ vs. the sham-treated group and ${ }^{\# \#} \mathrm{P}<0.01$ vs. the DM-treated group. THY, thymoquinone; TNF- $\alpha$, tumor necrosis- $\alpha$; DM, diabetes mellitus, IL-6, interleukin-6.

groups (Fig. 5A). Following treatment with thymoquinone, the heart rate levels of the DM + THY group were significantly reduced compared with the DM group $(\mathrm{P}<0.01$; Fig. 5A) However, BP levels did not exhibit a significant difference among the experimental groups (Fig. 5B).

Effect of thymoquinone treatment on oxidative stress levels. Following DM-induction in rats, MDA levels were significantly increased and SOD levels were reduced compared with the sham $(\mathrm{P}<0.01$; Fig. 6) and THY-treated groups (Fig. 6). Furthermore, administration of thymoquinone significantly reduced the oxidative stress damage in the DM rats, compared with the DM group without the treatment (P<0.01; Fig. 6).

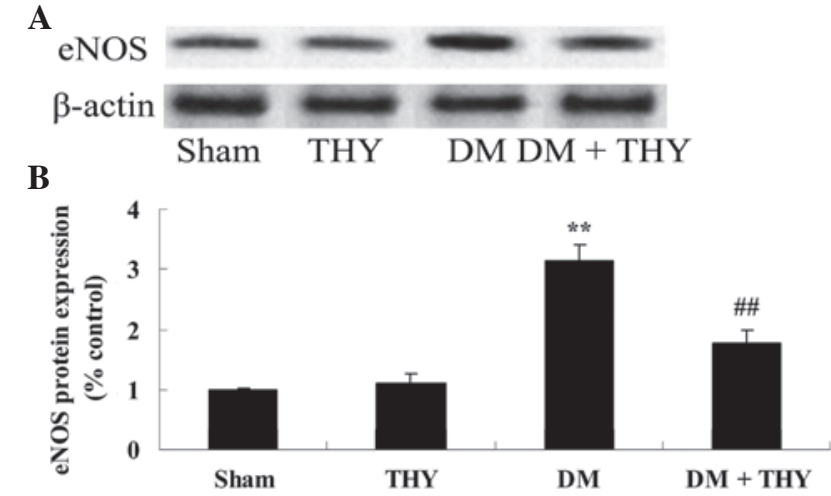

Figure 8. Effect of THY on the eNOS protein expression. (A) Western blotting assay and (B) statistical analysis of protein expression levels in the sham, THY-treated, DM-induced and DM + THY-treated groups. ${ }^{* *} \mathrm{P}<0.01$ vs. the sham-treated group and ${ }^{\# \#} \mathrm{P}<0.01$ vs. the DM-treated group. THY, thymoquinone; eNOS, endothelial nitric oxide synthase; DM, diabetes mellitus.

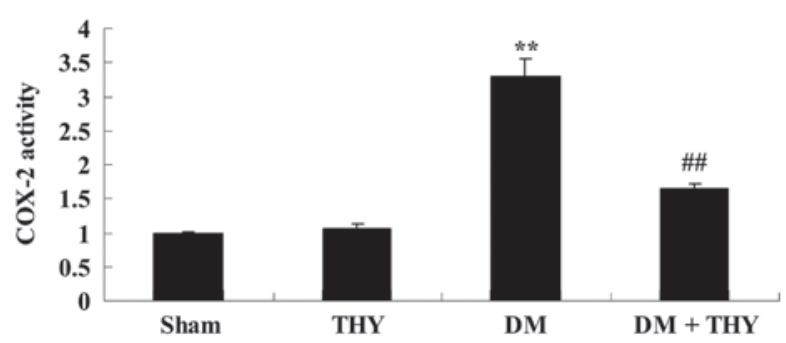

Figure 9. Effect of THY on the expression of COX-2 activity in the sham, THY-treated, DM-induced and DM + THY-treated groups. ${ }^{* *} \mathrm{P}<0.01$ vs. the sham-treated group and ${ }^{\# \#} \mathrm{P}<0.01$ vs. the DM-treated group. THY, thymoquinone; COX-2, cyclooxygenase-2; DM, diabetes mellitus.

Effect of thymoquinone treatment on TNF- $\alpha$ and IL- 6 inflammatory factors. As demonstrated in Fig. 7, TNF- $\alpha$ and IL-6 levels were significantly increased compared with the sham $(\mathrm{P}<0.01)$ and THY-treated groups. In addition, the levels of TNF- $\alpha$ and IL-6 in the DM-induced rats were observed to return to normal levels following treatment with thymoquinone, compared with the DM group $(\mathrm{P}<0.01 ;$ Fig. 7$)$.

Effect of thymoquinone treatmentonthe eNOSprotein expression levels. As demonstrated in Fig. 8, the eNOS protein expression levels were significantly increased in the DM group compared with the sham $(\mathrm{P}<0.01)$ and thymoquinone groups. In contrast, the increase of the eNOS protein expression levels was inhibited by treatment with thymoquinone in the DM + THY-treated group compared with the DM group $(\mathrm{P}<0.01$; Fig. 8).

Effect of thymoquinone treatment on COX-2 activity. As demonstrated in Fig. 9, the COX-2 levels in the DM group were markedly increased compared with the sham $(\mathrm{P}<0.01)$ and THY-treated groups. However, administration of thymoquinone in the DM + THY group indicated an inhibition of this effect compared with the DM group ( $\mathrm{P}<0.01$; Fig. 9).

Effect of thymoquinone treatment on caspase-3 activity. As demonstrated in Fig. 10, DM-induction increased caspase-3 


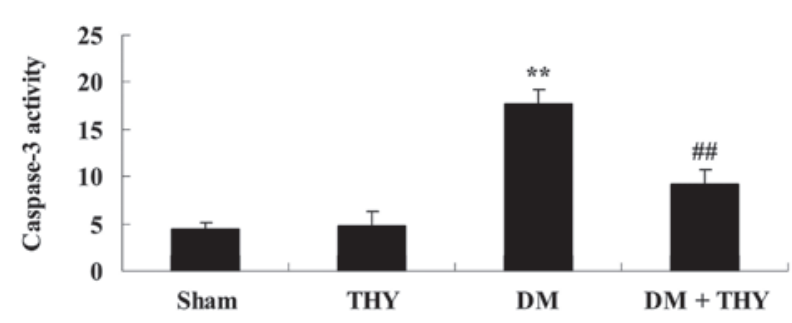

Figure 10. Effect of THY on the caspase- 3 activity in the sham, THY-treated, DM-induced and DM + THY-treated groups. ${ }^{* *} \mathrm{P}<0.01$ vs. the sham-treated group and ${ }^{\# \#} \mathrm{P}<0.01$ vs. the DM-treated group. THY, thymoquinone; DM, diabetes mellitus.

A

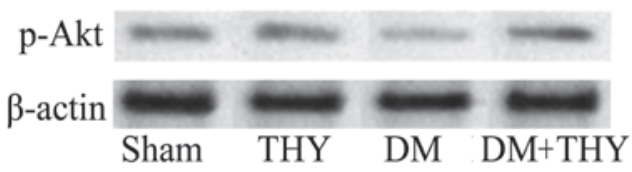

B

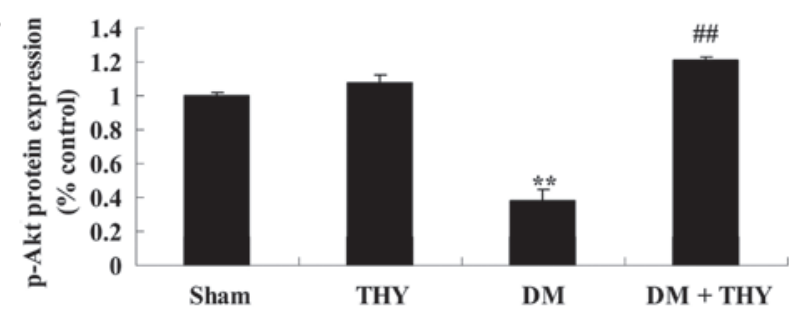

Figure 11. Effect of THY on p-Akt protein expression. (A) Western blotting assay and (B) statistical analysis of p-Akt in rats with diabetes mellitus. ${ }^{* * *} \mathrm{P}<0.01$ vs. the sham-treated group and ${ }^{\# \#} \mathrm{P}<0.01$ vs. the DM-treated group. THY, thymoquinone; p-Akt, phosphorylated-protein kinase B; DM, diabetes mellitus.

activity compared with the sham $(\mathrm{P}<0.01)$ and THY-treated groups. In contrast, thymoquinone treatment resulted in a significant reduction in caspase-3 activity in the DM $+\mathrm{THY}$ group compared with the DM group $(\mathrm{P}<0.01$; Fig. 10).

Effect of thymoquinone treatment on the $p$-Akt protein expression levels. A significant reduction in the p-Akt protein expression levels of DM rats was observed compared with the sham $(\mathrm{P}<0.01$; Fig. 11) and THY-treated groups (Fig. 11). Similarly, the reduction in the p-Akt levels was significantly suppressed by administration of thymoquinone in the $\mathrm{DM}+\mathrm{THY}$ group compared with the DM group $(\mathrm{P}<0.01$; Fig. 11).

\section{Discussion}

$\mathrm{DM}$ is a common and frequently occurring disease of the endocrine system resulting from the combined effect of several factors and characterized by alterations in the glucose metabolism (12). DCD is considered the main cause of mortality and disability in patients with DM and results in alterations in the pathology of multiple organs, including the kidney, eyes, heart and skin (13). The prevalence rate of DCD in patients with DM is $40 \%$ in the USA and Europe, which is 17 times higher than in patients without DM (14). The prevalence rate of DCD in China increases in line with age (15), and the prevalence is $>50 \%$ in individuals over 60 years old (16).
The present study demonstrated that thymoquinone treatment significantly increased the insulin levels and body weight, and reduced the blood glucose and heart rate levels in DM rats. El-Mahmoudy et al (17) indicated that thymoquinone protects against the STZ-induced DM via inhibition of nitric oxide. Furthermore, Pari and Sankaranarayanan (18) demonstrated that thymoquinone protects against hepatic dysfunction in STZ-nicotinamide-induced DM rats. The results of the present study were consistent with those of the previous studies, thus, thymoquinone may be an improved agent for DCD therapy.

Oxidative stress refers to the tissue damage resulting from the disequilibrium of the overproduction of reactive oxygen species (ROS) and the inhibition of the antioxidant defense mechanisms (19). A previous study suggested that the circulating levels of markers may increase due to ROS damage and the inability to prevent this effect (19). Patients with DM suffer from lipid disorders that lead to the production of ROS and oxidative stress $(19,20)$. The activation of glucose-induced eNOS may lead to endothelial dysfunction due to the production of oxidative stress (21). In the current study, pretreatment with thymoquinone significantly inhibited the MDA levels and increased SOD activity in DM rats. Farag et al (22) demonstrated that thymoquinone improves the disease pathology in injured kidney and liver tissue by resetting the oxidant/antioxidant balance of the affected organs in rats with acute renal ischemia/reperfusion. Mabrouk and Ben Cheikh (23) suggested that thymoquinone supplementation reversed lead-induced oxidative stress in adult rat testes. These results indicate that the effect of thymoquinone on DCD may be associated with the anti-oxidative effect observed in the DM rats of the current study.

IR and insulin secretory defects further contribute to the pathogenesis of DM, however the mechanisms underlying this remain to be fully understood (24). A previous study demonstrated that various inflammatory factors are associated with $\mathrm{DM}$, such as C-reactive protein (CRP), thus, inflammation may be the key initiator of IR (25). Cytokines are regulators of the adipose tissue metabolism and stimulating factors, such as overnutrition, may lead to an increase in the secretion of IL- 6 and TNF- $\alpha$ by the adipocytes. This may further lead to an increase in the hepatic synthesis and secretion of CRP (26). Furthermore, inhibition of insulin receptor tyrosine kinase activity and the aggravation of IR result in the production of macrophage migration inhibitory factor (MIF) (24). MIF can induce the production of TNF- $\alpha$, and their interaction may affect the balance of insulin levels, and lead to IR (24). Previous studies suggested that insulin may inhibit the synthesis of $\mathrm{CRP}$, as in the case of IR, the sensitivity of the liver to insulin is suppressed, leading to a rapid increase in the synthesis of CRP $(24,25)$. At present, evidence indicates that chronic inflammation serves an important role in the pathogenesis of IR, however the mechanism remains to be elucidated (24).

In the current study, thymoquinone treatment significantly suppressed the TNF- $\alpha$ and IL-6 levels in DM rats. Periyanayagam et al (27) observed that thymoquinone ameliorates NLRP3-induced inflammation in albino Wistar rats administered with ethanol and fed a high-fat diet. Rifaioglu et al (28) demonstrated that thymoquinone has an antioxidative and anti-inflammatory effect in an acute pseudomonas prostatitis rat model. These results indicated that the 
effect of thymoquinone on DCD may be associated with an anti-inflammatory effect in DM rat.

Endothelial progenitor cells (EPCs) are a type of precursors that differentiate into vascular endothelial cells (29). Previous studies demonstrated that EPCs are involved in the angiogenesis of human embryos and the repair process in endothelial injury $(29,30)$. EPCs have an important role in the occurrence and development of vascular complications in DM $(29,31)$. eNOS is the key enzyme that regulates the production of endothelium-derived nitric oxide (21) and has an effect on the phosphatidylinositol 3-kinase/Akt signaling pathway (32). The PI3K/Akt pathway regulates the metabolism, growth, migration and proliferation of various cells (32). The results obtained from the current study demonstrated that treatment with thymoquinone significantly reduced the eNOS protein expression levels, COX-2 and caspase-3 activity levels, and increased the p-Akt protein expression levels in the DM rats. Idris-Khodja and Schini-Kerth (33) determined that thymoquinone improves the aging-associated endothelial dysfunction through eNOS in the rat mesenteric artery. Kundu et al (34) demonstrated that in vivo thymoquinone treatment inhibits the phorbol ester-induced NF- $\mathrm{KB}$ and COX-2 expression levels in the skin from mice. Yu and Kim (35) suggested that thymoquinone suppressed COX-2 and caspase-3 activity levels and activated the PI3K/Akt pathway in rabbit articular chondrocytes. The effect of thymoquinone on DCD is suggested to be associated with the downregulation of eNOS, COX-2 and caspase-3 activity levels and the upregulation of PI3K/Akt pathway.

In conclusion, the present study demonstrated that the protective effect of thymoquinone improves the cardiovascular function through antioxidative, anti-inflammatory and anti-apoptotic effects in DM rats. The PI3K/Akt pathway is a possible target in future research focusing upon treatment with thymoquinone to protect cardiovascular function of DM rats.

\section{References}

1. Yin L, Cai WJ, Chang XY, Li J, Su XH, Zhu LY, Wang XL and Sun K: Association between fetuin-A levels with insulin resistance and carotid intima-media thickness in patients with new-onset type 2 diabetes mellitus. Biomed Rep 2: 839-842, 2014.

2. Cheung KK, Luk AO, So WY, Ma RC, Kong AP, Chow FC and Chan JC: Testosterone level in men with type 2 diabetes mellitus and related metabolic effects: A review of current evidence. J Diabetes Investig 6: 112-123, 2015.

3. Li MZ, Su L, Liang BY, Tan JJ, Chen Q, Long JX, Xie JJ, Wu GL, Yan Y, Guo XJ and Gu L: Trends in prevalence, awareness, treatment, and control of diabetes mellitus in mainland china from 1979 to 2012. Int J Endocrinol 2013: 753150, 2013.

4. Guo J, Whittemore R and He GP: The relationship between diabetes self-management and metabolic control in youth with type 1 diabetes: An integrative review. J Adv Nurs 67: 2294-2310, 2011.

5. Peng D, Wang J, Zhang R, Tang S, Jiang F, Chen M, Yan J, Sun X, Wang T, Wang S, et al : C-reactive protein genetic variant is associated with diabetic retinopathy in Chinese patients with type 2 diabetes. BMC Endocr Disord 15: 8, 2015.

6. Huang CQ, Ma GZ, Tao MD, Ma XL, Feng J and Liu QX: The relationship between renal injury and change in vitamin $\mathrm{D}$ metabolism in aged rats with insulin resistance or type 2 diabetes mellitus. J Int Med Res 36: 289-295, 2008.

7. Göbl CS, Brannath W, Bozkurt L, Handisurya A, Anderwald C, Luger A, Krebs M, Kautzky-Willer A and Bischof MG: Sex-specific differences in glycemic control and cardiovascular risk factors in older patients with insulin-treated type 2 diabetes mellitus. Gend Med 7: 593-599, 2010
8. Rutter MK and Nesto RW: Ischemia imaging and plaque imaging in diabetes: Complementary tools to improve cardiovascular risk management: Response to Raggi et al. Diabetes Care 29: 1187, author reply 1188,2006

9. Aycan IO, Tokgöz O, Tüfek A, Alabalık U, Evliyaoğlu O, Turgut H, Çelik F and Güzel A: The use of thymoquinone in nephrotoxicity related to acetaminophen. Int J Surg 13: 33-37, 2015.

10. Khan MA, Anwar S, Aljarbou AN, Al-Orainy M, Aldebasi YH, Islam $\mathrm{S}$ and Younus $\mathrm{H}$ : Protective effect of thymoquinone on glucose or methylglyoxal-induced glycation of superoxide dismutase. Int J Biol Macromol 65: 16-20, 2014.

11. Han J, Wang LU, Bian H,Zhou X and Ruan C: Effects of paroxetine on spatial memory function and protein kinase $\mathrm{C}$ expression in a rat model of depression. Exp Ther Med 10: 1489-1492, 2015.

12. Cabrera SM, Rigby MR and Mirmira RG: Targeting regulatory $\mathrm{T}$ cells in the treatment of type 1 diabetes mellitus. Curr Mol Med 12: 1261-1272, 2012.

13. De la Sierra A: Angiotensin receptor blockers in hypertension and cardiovascular diseases. Cardiovasc Hematol Agents Med Chem 4: 67-73, 2006.

14. Hassan MH and Abd-Allah GM: Effects of metformin plus gliclazide versus metformin plus glimepiride on cardiovascular risk factors in patients with type 2 diabetes mellitus. Pak J Pharm Sci 28: 1723-1730, 2015.

15. Trost S, Pratley R and Sobel B: Impaired fibrinolysis and risk for cardiovascular disease in the metabolic syndrome and type 2 diabetes. Curr Diab Rep 6: 47-54, 2006.

16. Forst T, Anastassiadis E, Diessel S,Löffler A and Pfützner A: Effect of linagliptin compared with glimepiride on postprandial glucose metabolism, islet cell function and vascular function parameters in patients with type 2 diabetes mellitus receiving ongoing metformin treatment. Diabetes Metab Res Rev 30: 582-589, 2014.

17. El-Mahmoudy A, Shimizu Y, Shiina T, Matsuyama H,El-Sayed M and Takewaki T: Successful abrogation by thymoquinone against induction of diabetes mellitus with streptozotocin via nitric oxide inhibitory mechanism. Int Immunopharmacol 5: 195-207, 2005.

18. Pari L and Sankaranarayanan C: Beneficial effects of thymoquinone on hepatic key enzymes in streptozotocin-nicotinamide induced diabetic rats. Life Sci 85: 830-834, 2009.

19. Li X, Zhao H, Wang Q, Liang H and Jiang X: Fucoidan protects ARPE-19 cells from oxidative stress via normalization of reactive oxygen species generation through the $\mathrm{Ca}^{2+}$-dependent ERK signaling pathway. Mol Med Rep 11: 3746-3752, 2015.

20. Matsunami T, Sato Y, Sato T, Ariga S, Shimomura T and Yukawa M: Oxidative stress and gene expression of antioxidant enzymes in the streptozotocin-induced diabetic rats under hyperbaric oxygen exposure. Int J Clin Exp Pathol 3: 177-188, 2009.

21. Huang A, Yang YM, Feher A, Bagi Z, Kaley G and Sun D: Exacerbation of endothelial dysfunction during the progression of diabetes: Role of oxidative stress. Am J Physiol Regul Integr Comp Physiol 302: R674-R681, 2012.

22. Farag MM, Ahmed GO, Shehata RR and Kazem AH: Thymoquinone improves the kidney and liver changes induced by chronic cyclosporine A treatment and acute renal ischaemia/reperfusion in rats. J Pharm Pharmacol 67: 731-739, 2015.

23. Mabrouk A and Ben Cheikh H: Thymoquinone supplementation reverses lead-induced oxidative stress in adult rat testes. Gen Physiol Biophys 34: 65-72, 2015.

24. Yin L, Cai WJ, Zhu LY, Li J, Su XH, Wang XL, Chang XY and Sun K: Association of plasma Fetuin-A and clinical characteristics in patients with new-onset type 2 diabetes mellitus. Int J Clin Exp Med 8: 991-999, 2015.

25. Javed F, Al-Kheraif AA, Salazar-Lazo K, Yanez-Fontenla V, Aldosary KM, Alshehri M, Malmstrom H and Romanos GE: Periodontal inflammatory conditions among smokers and never-smokers with and without Type 2 diabetes mellitus. J Periodontol 86: 839-846, 2015.

26. Schäffler A, Zeitoun M, Wobser H, Buechler C, Aslanidis C and Herfarth $\mathrm{H}$ : Frequency and significance of the novel single nucleotide missense polymorphism Val109Asp in the human gene encoding omentin in Caucasian patients with type 2 diabetes mellitus or chronic inflammatory bowel diseases. Cardiovasc Diabetol 6: 3, 2007.

27. Periyanayagam S, Arumugam G, Ravikumar A and Ganesan VS: Thymoquinone ameliorates NLRP3-mediated inflammation in the pancreas of albino Wistar rats fed ethanol and high-fat diet. J Basic Clin Physiol Pharmacol 26: 623-632, 2015 . 
28. Rifaioglu MM, Nacar A, Yuksel R, Yonden Z, Karcioglu M Zorba OU, Davarci I and Sefil NK: Antioxidative and anti-inflammatory effect of thymoquinone in an acute Pseudomonas prostatitis rat model. Urol Int 91: 474-481, 2013.

29. Sun N, Wang H and Wang L: Vaspin alleviates dysfunction of endothelial progenitor cells induced by high glucose via PI3K/Akt/eNOS pathway. Int J Clin Exp Pathol 8: 482-489, 2015.

30. Sorrentino SA, Bahlmann FH, Besler C, Müller M, Schulz S, Kirchhoff N, Doerries C, Horváth T, Limbourg A, Limbourg F, et al: Oxidant stress impairs in vivo reendothelialization capacity of endothelial progenitor cells from patients with type 2 diabetes mellitus: Restoration by the peroxisome proliferator-activated receptor-gamma agonist rosiglitazone. Circulation 116: 163-173, 2007.

31. Tang Y, Jacobi A, Vater C, Zou X and Stiehler M: Salvianolic acid $\mathrm{B}$ protects human endothelial progenitor cells against oxidative stress-mediated dysfunction by modulating Akt/mTOR/4EBP1, p38 MAPK/ATF2, and ERK1/2 signaling pathways. Biochem Pharmacol 90: 34-49, 2014.
32. Wang T, Mao X, Li H, Qiao S, Xu A, Wang J, Lei S, Liu Z, $\mathrm{Ng} \mathrm{KF}$, Wong GT, et al : N-Acetylcysteine and allopurinol up-regulated the Jak/STAT3 and PI3K/Akt pathways via adiponectin and attenuated myocardial postischemic injury in diabetes. Free Radic Biol Med 63: 291-303, 2013.

33. Idris-Khodja $\mathrm{N}$ and Schini-Kerth V: Thymoquinone improves aging-related endothelial dysfunction in the rat mesenteric artery. Naunyn Schmiedebergs Arch Pharmacol 385: 749-758, 2012.

34. Kundu JK, Liu L, Shin JW and Surh YJ: Thymoquinone inhibits phorbol ester-induced activation of $\mathrm{NF}-\kappa \mathrm{B}$ and expression of COX-2, and induces expression of cytoprotective enzymes in mouse skin in vivo. Biochem Biophys Res Commun 438: 721-727, 2013.

35. Yu SM and Kim SJ: The thymoquinone-induced production of reactive oxygen species promotes dedifferentiation through the ERK pathway and inflammation through the p38 and PI3K pathways in rabbit articular chondrocytes. Int J Mol Med 35: 325-332, 2015. 